\title{
Time development of small disturbances to plane Couette Flow
}

Article

Published Version

Shepherd, T. G. (1985) Time development of small disturbances to plane Couette Flow. Journal of the Atmospheric Sciences, 42 (17). pp. 1868-1872. ISSN 15200469 doi: https://doi.org/10.1175/15200469(1985)042<1868:TDOSDT>2.0.CO;2 Available at https://centaur.reading.ac.uk/32996/

It is advisable to refer to the publisher's version if you intend to cite from the work. See Guidance on citing.

Published version at: http://dx.doi.org/10.1175/1520-0469(1985)042<1868:TDOSDT>2.0.CO;2

To link to this article DOI: http://dx.doi.org/10.1175/1520-

0469(1985)042<1868:TDOSDT>2.0.CO;2

Publisher: American Meteorological Society

All outputs in CentAUR are protected by Intellectual Property Rights law, including copyright law. Copyright and IPR is retained by the creators or other copyright holders. Terms and conditions for use of this material are defined in the End User Agreement.

www.reading.ac.uk/centaur 
Central Archive at the University of Reading

Reading's research outputs online 


\title{
Time Development of Small Disturbances to Plane Couette Flow
}

\author{
THEODORE G. SHEPHERD \\ Department of Applied Mathematics and Theoretical Physics, University of Cambridge, Cambridge CB3 9EW, England
}

(Manuscript received 2 January 1985, in final form 1 April 1985)

\begin{abstract}
The question of linear sheared-disturbance evolution in constant-shear parallel flow is here reexamined with regard to the temporary-amplification phenomenon noted first by Orr in 1907. The results apply directly to Rossby waves on a beta-plane, and are also relevant to the Eady model of baroclinic instability. It is shown that an isotropic initial distribution of standing waves maintains a constant energy level throughout the shearing process, the amplification of some waves being precisely balanced by the decay of the others. An expression is obtained for the energy of a distribution of disturbances whose wavevectors lie within a given angular wedge, and an upper bound derived. It is concluded that the case for ubiquitous amplification made in recent studies may have been somewhat overstated: while carefully-chosen individual Fourier components can amplify considerably before they decay, a general distribution will tend to exhibit little or no amplification.
\end{abstract}

\section{耳. Introduction}

The subject of the time development of sheared disturbances has a long and interesting history, dating back at least as far as Lord Kelvin (Thomson, 1887). In the course of addressing certain questions regarding the stability of shear flow, Kelvin made the important contribution of finding an exact solution to the problem of disturbance development in two-dimensional viscous parallel flow with constant shear. This early work was then developed further by Orr (1907), who resolved many of the stability issues by clarifying the distinction between the discrete (or normal-mode) spectrum and the continuous spectrum of perturbation eigenfunctions. Then by considering the evolution of arbitrary initial disturbances in the manner of Kelvin, Orr showed that the energy density of inviscid plane waves with phase lines leaning into the shear would experience a temporary amplification before eventually dying away. He summarized this phenomenon as follows: "It accordingly appears that, in this simple case, although the disturbance, if sufficiently small [(so that linear theory is valid - a non sequitur for single plane waves which happen to be exact nonlinear solutions)], must ultimately decrease indefinitely, yet, before doing so, it may be very much increased . . . [Indeed] the ratio of increase may be made as great as we like." (Orr, 1907, p. 32; bracketed text mine.)

Within the geophysical fluid dynamics community, it is fair to say that the striking normal-mode baroclinic instability theories of Charney (1947) and of Eady (1949) served to divert the attention of most researchers away from the study of initial-value problems. Indeed it is only fairly recently that the insights of Kelvin and Orr have been actively revived in the literature, in reexaminations of the plane Couette flow problem $U(y)$ = Sy by Rosen (1971), Yamagata (1976), Farrell (1982), Tung (1983), and Boyd (1983). While the last four authors allow the possibility of Rossby wave propagation, this does not alter the nature of the problem in any fundamental way: propagating waves always encounter the same shear $S$. Predating these recent studies are the papers of Case (1960) and of Pedlosky (1964), but those analyses are valid only in the asymptotic limit of large time; they consequently miss the temporary amplification phenomenon which is the focus of current attention.

The purpose of the present note is to point out a few aspects of the plane Couette flow problem that have not yet been described in the literature, particularly with regard to the extent of temporary amplification. To keep the treatment as simple as possible and to facilitate direct comparison with most of the recent work, attention is limited to the case of inviscid disturbances in an unbounded linear shear flow. The exact solution of the linearized initial-value problem is described in Section 2, while the energetic evolution of a single standing wave is derived in Section 3. The new results come from considering the integrated evolution of a distribution of initial disturbances, in Section 4; the principal finding is that an initially isotropic distribution maintains a constant energy level for all time. In consequence, an initial distribution of standing waves of uniform amplitude with wavevector angles covering a section $\Delta \theta$ out of $\pi / 2$ has its amplification bounded by $\pi /(2 \Delta \theta)$. This suggests that the case for ubiquitous amplification made in recent studies may have been somewhat overstated. Some of the implications of these results are discussed in Section 5. 


\section{The exact solution}

The inviscid linear evolution of disturbances in a shear flow is governed by the linearized vorticity equation

$$
\left(\frac{\partial}{\partial t}+U \frac{\partial}{\partial x}\right) \nabla^{2} \psi-U_{y y} \psi_{x}=0
$$

where $\psi$ is the disturbance streamfunction and $U(y, t)$ the given basic-state flow. In general, the nonseparable form of (1) prevents an exact analytical solution. For a linear shear flow $U=S y$, however, (1) may be manipulated into a separable equation in the spatial coordinates by using the "convected coordinates" transformation

$$
\xi=x-S y t ; \quad \eta=y ; \quad \tau=t .
$$

This procedure was employed implicitly by both Kelvin (Thomson, 1887) and Orr (1907), and formalized by Phillips (1966). Using (2) to transform (1), the material derivative becomes $\partial / \partial \tau$ and one obtains

$$
\frac{\partial}{\partial \tau} \zeta \equiv \frac{\partial}{\partial \tau}\left[\frac{\partial^{2}}{\partial \xi^{2}}+\left(\frac{\partial}{\partial \eta}-S \tau \frac{\partial}{\partial \xi}\right)^{2}\right] \psi=0,
$$

where the vorticity $\zeta=\psi_{x x}+\psi_{y y}$ and is evidently conserved; Eq. (3) must be considered together with the initial condition $\zeta(\xi, \eta, \tau=0)=\zeta_{0}(\xi, \eta)$ and the boundary condition that $|\zeta|$ remain bounded as $\xi, \eta \rightarrow \infty$. Then (3) allows the exact, $\tau$-independent solution

$$
\zeta(\xi, \eta, \tau)=\operatorname{Re}\left[\hat{\zeta}_{0}(k, l) e^{i(k \xi+l \eta)}\right],
$$

where $\hat{\zeta}_{0}(k, l)$ is the Fourier transform of $\zeta_{0}(\xi, \eta)$. The principle of superposition being valid for this linear problem, one can build up the solution to general initial conditions by combining plane waves of the form (4); in the original coordinates these become

$$
\zeta(x, y, t)=\operatorname{Re}[\exp i[k x+(l-S k t) y+\phi]],
$$

where $\phi$ is a phase shift parameter. It is evident that the solution (5) is nonseparable, but that at any given time it represents a plane wave. Indeed the time evolution of the wave is simply that of a tilting over of phase lines towards the $x$-axis, sometimes called the "Venetian-blind effect."

The relation between $\psi$ and $\zeta$, together with the conservation of wave enstrophy density, both described by (3), lead to the following time dependence for the wave (kinetic) energy density:

$$
\begin{aligned}
E(k, l, t) & =\frac{\frac{1}{2}|\zeta|^{2}}{k^{2}+(l-S k t)^{2}} \\
& =\frac{k^{2}+l^{2}}{k^{2}+(l-S k t)^{2}} E(k, l, 0),
\end{aligned}
$$

written as a function of the initial wavenumbers $k$ and $l$. Incidentally, this expression is identical to those obtained by Tung (1983) and by Boyd (1983) in their Rossby-wave analyses, demonstrating the irrelevance of beta to this aspect of the problem. The properties of (6) are strikingly evident: the energy of any wave with $k \neq 0$ will eventually decay with a $t^{-2}$ dependence; but if $S k l>0$, then there will be a temporary amplification preceding the decay. It is the latter point which has attracted so much recent interest, and which was raised originally by Orr (1907). The former feature is the one which is relevant to asymptotic analyses such as those of Case (1960) and of Pedlosky (1964); it was also discussed by Kelvin (Thomson, 1887).

\section{Temporary amplification of a standing wave}

In what follows the analysis is limited to the standingwave problem, namely, that beginning from a pair of waves $(k, l)$ and $(k,-l)$ with the same initial energy. Since the interest is in integrated results, this is not really restrictive. Standing waves have also been explicitly treated by Orr (1907) and by Boyd (1983). One may choose $k$ and $S$ positive for definiteness, and then consideration of positive $l$ covers the range of possible waves. There is no loss of generality in scaling time so that $S=1$, and length so that $k^{2}+l^{2}=1$. Then the ratio of actual to initial wave energy density of the two waves may be written

$$
\begin{aligned}
I(k, l, t) & \equiv \frac{E(k, l, t)+E(k,-l, t)}{E(k, l, 0)+E(k,-l, 0)} \\
& =\frac{1+k^{2} t^{2}}{\left(1+k^{2} t^{2}\right)^{2}-4 k^{2} l^{2} t^{2}} ;
\end{aligned}
$$

it is understood that $k, l \in[0,1]$ and $t \in[0, \infty)$. Now, to investigate if and when $I$ has a maximum, note that the condition for $\partial I / \partial t$ to vanish is that

$$
2 k^{6} t^{5}+4 k^{4} t^{3}+2 k^{2}\left(4 k^{2}-3\right) t=0,
$$

so that

$$
k t=0 \quad \text { or } \quad k t=(2 l-1)^{1 / 2} \text {; }
$$

in the above, the scaling relation $k^{2}+l^{2}=1$ has been used freely. The initial conditions guarantee that, with the exception of the trivial case $k=0$, the total wave field will initially have an amplifying component due to the $(k, l)$ contribution, as well as a decaying component due to the $(k,-l)$ contribution. Equation (9) states that at $t=0$ the instantaneous growth rate of the former will exactly balance the decay rate of the latter. As $t \rightarrow \infty$, of course, $I$ approaches zero. Whether the amplifying component can induce a temporary increase in $I$ evidently depends on the initial wavevector orientation: (9) implies that for $2 l<1$, or $\theta \equiv \arctan (l)$ $k)<\pi / 6, I(t)$ will decay monotonically; while if $\pi / 6$ $<\theta<\pi / 2$, then $I(t)$ will take a simple maximum. In the latter circumstance, it is readily verified that

$$
I_{\max }(l)=\frac{1}{4 l(1-l)} \text { for } \frac{1}{2} \leqslant l<1 .
$$

As $\theta \rightarrow \pi / 2$ and $l \rightarrow 1$, the maximum value of $I$ becomes arbitrarily large (the point of the Orr (1907) 
quotation given in Section 1), but it is attained after a very long time. In practice one might expect there to exist a minimum nonzero value of $k$ (through discretization of the spectrum in a numerical model, for example), in which case (10) would have an upper bound. But it is usually the moderate- $k$ disturbances which are of most interest, since they attain their maximum amplitude quite rapidly. For the $l=2 k \approx 0.89$ waves considered by Tung (1983) and by Boyd (1983), the maximum amplification $I$ is 2.65 and is attained at $t=2.0$.

\section{Integrated results}

As the single standing-wave initial conditions of Section 3 are somewhat special and rather unrealistic, it is of interest to examine the behavior of a distribution of initial waves. To arrive at a general formula, let $\theta \equiv \arctan (l / k)$ and define

$$
J\left(\theta_{1}, \theta_{2}, t\right) \equiv \frac{1}{\left(\theta_{2}-\theta_{1}\right)} \int_{\theta_{1}}^{\theta_{2}} I(\theta, t) d \theta
$$

to be the average amplification of waves with $\theta_{1} \leqslant \theta$ $\leqslant \theta_{2}$. Then substituting (7) with $k=\cos \theta$ and $l=\sin \theta$ into ( 11 ) yields

$$
\begin{aligned}
& J\left(\theta_{1}, \theta_{2}, t\right) \\
& \quad=\frac{1}{\left(\theta_{2}-\theta_{1}\right)} \int_{\theta_{1}}^{\theta_{2}} \frac{\left(1+t^{2} \cos ^{2} \theta\right) d \theta}{\left(1+t^{2} \cos ^{2} \theta\right)^{2}-4 t^{2} \cos ^{2} \theta \sin ^{2} \theta},
\end{aligned}
$$

which becomes, upon using the Euler substitution $x=\tan \theta$ (noting that $d \theta=\cos ^{2} \theta d x$ and that $\sec ^{2} \theta$ $\left.=1+x^{2}\right)$

$$
\begin{aligned}
J\left(\theta_{1}, \theta_{2}, t\right) & =\frac{1}{\left(\theta_{2}-\theta_{1}\right)} \int_{\tan \theta_{1} \cdot \tan \theta_{2}}^{\left.\tan x^{2}+t^{2}+2 x t\right)\left(1+x^{2}+t^{2}-2 x t\right)} \\
& =\frac{1}{2\left(\theta_{2}-\theta_{1}\right)} \int_{\tan \theta_{1}}^{\tan \theta_{2}}\left[\frac{1}{(x+t)^{2}+1}+\frac{1}{(x-t)^{2}+1}\right] d x \\
& =\frac{1}{2\left(\theta_{2}-\theta_{1}\right)}\left[\arctan \left(\tan \theta_{2}+t\right)+\arctan \left(\tan \theta_{2}-t\right)-\arctan \left(\tan \theta_{1}+t\right)-\arctan \left(\tan \theta_{1}-t\right)\right]
\end{aligned}
$$

The most striking new result comes from evaluating (12) with $\theta_{1}=0$ and $\theta_{2}=\pi / 2$, namely, for an average over all possible waves with $k^{2}+l^{2}=1$ :

$$
J(0, \pi / 2, t) \equiv 1 \text {. }
$$

Therefore the energy of an ensemble of waves with an initially isotropic distribution of energy remains constant for all time. This relation implies that the measure or weight of the amplifying waves is always large enough to balance the asymptotic decay of the others, yet is never so large that the total energy is greater than its initial value.

Consider the case of $\theta_{2}=\pi / 2-\epsilon$ with $\epsilon$ small, so that a small range of the shallowest waves is excluded, and $\theta_{1}=0$ as above; then (12) implies

$$
\begin{aligned}
& J\left(0, \frac{\pi}{2}-\epsilon, t\right) \\
& \approx \frac{1}{\pi}\left[\arctan \left(\frac{1+\epsilon t}{\epsilon}\right)+\arctan \left(\frac{1-\epsilon t}{\epsilon}\right)\right] \\
& \approx \begin{cases}1 & \text { for } \epsilon t \ll 1 \\
\frac{2}{\pi \epsilon t^{2}} & \text { for } \epsilon t \gg 1 ;\end{cases}
\end{aligned}
$$

hence the energy stays level until $t=\mathrm{O}(1 / \epsilon)$, and then drops off like $t^{-2}$ just as in the single-wave case. On the other hand, if $\theta_{2}=\pi / 2$ but $\theta_{1} \neq 0$, the fact that $J(0$, $\left.\theta_{1}, t\right) \rightarrow 0$ as $t \rightarrow \infty$ implies that

$$
J\left(\theta_{1}, \pi / 2, t\right) \rightarrow \frac{\pi}{\pi-2 \theta_{1}} \text { as } t \rightarrow \infty .
$$

For such distributions the asymptotic-decay fate of single plane waves is avoided. It is indeed easy to see from (13) that in general $J$ has the upper bound

$$
J\left(\theta_{1}, \theta_{2}, t\right) \leqslant \frac{\pi}{2\left(\theta_{2}-\theta_{1}\right)},
$$

but this is a least upper bound only when $\theta_{2}=\pi / 2$; otherwise the amplification falls short of (16) and the asymptotic decay eventually prevails.

Some plots of $J$ against time are shown in Fig. 1 for various values of $\theta_{1}$ and $\theta_{2}$, in order to illustrate the points discussed above. Even in the rather extreme case of $\theta_{1}=\pi / 3$ and $\theta_{2}=5 \pi / 12$ (or $\theta=60^{\circ}-75^{\circ}$ ) the amplification is only threefold, one-half of the bound (16). The physical point to be made is that it is rather unlikely that the energy of an arbitrary disturbance would be concentrated in a narrow range of wavevectors, still less in a single Fourier component: generally the measured energy would be spread over a fairly broad range of $\theta$, making the amplification process inefficient; moreover, the broader the range the smaller the bound (16) would become, and the more of an overestimate it would be.

\section{Discussion}

The much studied problem of the time development of sheared disturbances in plane Couette flow, solved originally by Lord Kelvin (Thomson, 1887), has been reexamined with special regard to Orr's (1907) temporary-amplification phenomenon. The results are directly applicable to the development of Rossby waves in linear shear flow, and, with the exception of bound- 


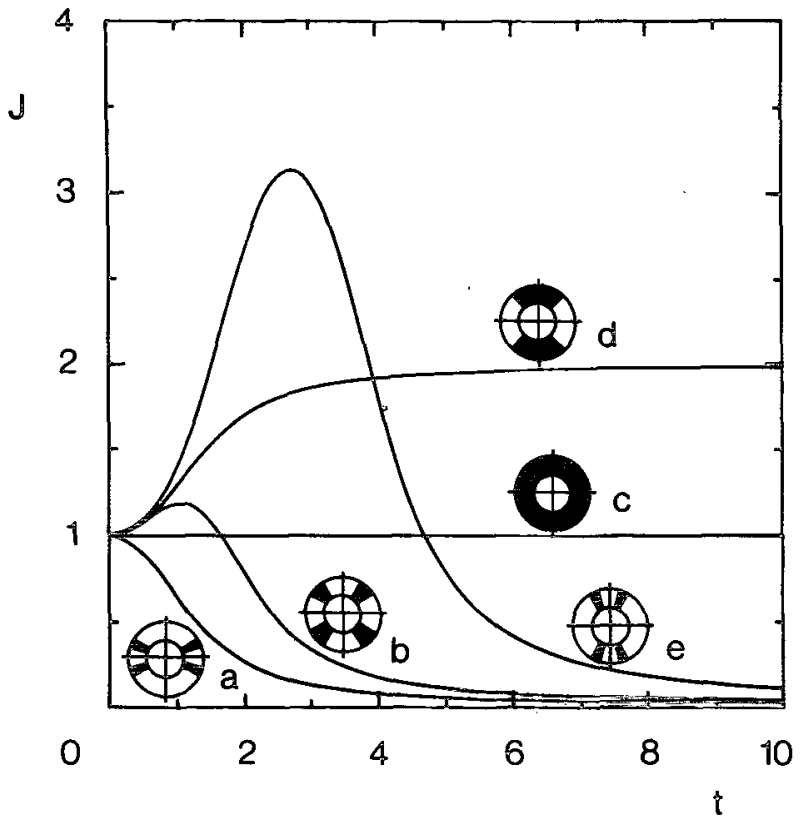

FIG. 1. The time evolution of $J\left(\theta_{1}, \theta_{2}, t\right)$ for selected values of $\theta_{1}$ and $\theta_{2}$ : (a) $15-30^{\circ}$; (b) $30-60^{\circ}$; (c) $0-90^{\circ}$; (d) $45-90^{\circ}$; (e) $60-75^{\circ}$. The small figures illustrate that portion of the spectral circle $k^{2}+l^{2}$ $=1$ which is included, with $k$ along the horizontal and $l$ along the vertical.

ary effects, to the Eady model of baroclinic instability. Temporary amplification of sheared disturbances, representing the initial algebraic growth by elements of the continuous spectrum, has been the object of much recent attention in the literature (e.g., Yamagata, 1976; Farrell, 1982; Tung, 1983; Boyd, 1983; and, in a somewhat more general context, Pierrehumbert, 1983). In fact, Farrell has gone so far as to suggest that this phenomenon might explain some cases of "Type B" cyclogenesis, in the sense defined by Petterssen and Smebye (1971). While Orr's result-namely, that the energetic amplification of a single plane wave may be made arbitrarily large by making the wave sufficiently shallow, i.e., $k \ll l$-is certainly true, the present work demonstrates that, for a general disturbance, one cannot expect any significant amplification whatsoever. Specifically, an isotropic initial distribution of waves with the same total wavenumber will maintain a constant energy level for all time.

Corollaries of the foregoing result follow easily. Any initial distribution of standing waves of uniform amplitude which includes the region about $k=0$ will grow monotonically, approaching a large-time asymptotic limit of an amplification factor equal to the inverse of the fraction of the spectral band originally excited [see (15)], and avoiding the single-wave asymptotic decay. For any such distribution which excludes the immediate vicinity of $k=0$ the same upper bound must apply, but it may no longer be a very good indicator of the maximum amplitude; indeed the energy need not grow at all. Even if the energy does grow, the bound will be quite severe for a distribution with reasonably large spectral breadth, and the amplification could well be insignificant.

The connection between idealized studies such as the present one-which is linearized, zonally homogeneous, and restricted to a rather special mean-flow shear-and the observed development of atmospheric disturbances, is, to say the least, tenuous. However, it is perhaps no more tenuous than the presumed connection for normal-mode instability calculations. The most promising avenue of future research into "development theory" must surely be the consideration of the evolution of unstable disturbances in zonallyinhomogeneous flows, with nonlinear wave, mean-flow interaction effects included; unfortunately, the mathematics involved in such investigations is extremely forbidding. The present work illustrates that even the simplest, most resolved problems may have surprising characteristics yet to be elucidated, the discovery of which is essential to an understanding of more complicated phenomena.

Acknowledgments. The author wishes to thank Dr. M. E. McIntyre for his encouragement and advice concerning the presentation of this material. The research has been supported through a Postdoctoral Fellowship from the Natural Sciences and Engineering Research Council of Canada.

\section{REFERENCES}

Boyd, J. P., 1983: The continuous spectrum of linear Couette flow with the beta effect. J. Atmos. Sci., 40, 2304-2308.

Case, K. M., 1960: Stability of inviscid plane Couette flow. Phys. Fluids, 3, 143-148.

Charney, J. G., 1947: The dynamics of long waves in a baroclinic westerly current. $J$. Meteor., 4, 135-162.

Eady, E. T., 1949: Long waves and cyclone waves. Tellus, 1, 33-52.

Farrell, B. F., 1982: The initial growth of disturbances in a baroclinic flow. J. Atmos. Sci., 39, 1663-1686.

Orr, W. M' F., 1907: The stability or instability of the steady motions of a perfect liquid and of a viscous liquid. Parts I and II. Proc. Roy. Irish Acad., A27, 9-68; 69-138.

Pedlosky, J., 1964: An initial value problem in the theory of baroclinic instability. Tellus, $16,12-17$.

Petterssen, S., and S. J. Smebye, 1971: On the development of extratropical cyclones. Quart. J. Roy. Meteor. Soc., 97, 457-482.

Phillips, O. M., 1966: The Dynamics of the Upper Ocean, 1st ed., Cambridge University Press, 178-185.

Pierrehumbert, R. T., 1983: Bounds on the growth of perturbations to nonparallel steady flow on the barotropic beta-plane. J. Atmos. Sci., 60, 1207-1217.

Rosen, G., 1971: General solution for perturbed plane Couette flow. Phys. Fluids, 14, 2767-2769.

Thomson, W., 1887: Stability of fluid motion-Rectilineal motion of viscous fluid between two parallel planes. Phil. Mag., 24, 188-196.

Tung, K. K., 1983: Initial-value problems for Rossby waves in a shear flow with critical level. J. Fluid Mech., 133, 443-469.

Yamagata, T., 1976: On trajectories of Rossby wave-packets released in a lateral shear flow. J. Ocean-Soc. Japan, 32, 162-168. 